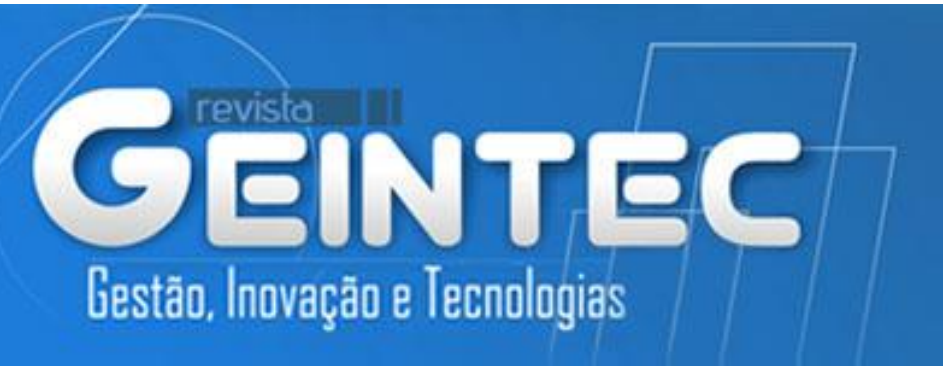

\title{
PROSPECÇÃO TECNOLÓGICA DO JABORANDI (Pilocarpus microphyllus): ESPÉCIE ECONOMICAMENTE IMPORTANTE NO NORTE E NORDESTE DO BRASIL
}

\section{TECHNOLOGICAL FORECASTING OF JABORANDI (Pilocarpus microphyllus): ECONOMICALLY IMPORTANT SPECIE IN THE NORTH AND NORTHEAST OF BRAZIL}

David Fernandes Lima ${ }^{1}$; Rogério Almiro Oliveira Silva ${ }^{2}$, Lana Grasiela Alves Marques ${ }^{3}$; Leiz Maria Costa Véras ${ }^{1}$; Evelyne Rolim Braun Simões ${ }^{5}$; José Roberto de Souza de Almeida Leite ${ }^{1}$; Maria Rita de Morais Chaves Santos ${ }^{7}$; Cláudia Pessoa ${ }^{8}$

${ }^{1}$ Núcleo de Pesquisa em Biodiversidade e Biotecnologia, BIOTEC. Rede Nordeste de Biotecnologia Renorbio -Universidade Federal do Piauí - UFPI - Parnaíba/PI - Brasil

david@ vegeflora.com.br; leiz.veras@gmail.com; jrsaleite@gmail.com

${ }^{2}$ Rede Nordeste de Biotecnologia - Renorbio -Universidade Federal do Piauí - UFPI - Teresina/PI - Brasil rogerio.ufpi@gmail.com

${ }^{5}$ Rede Nordeste de Biotecnologia - Renorbio - Universidade Federal do Ceará - UFC

Fortaleza/CE - Brasil

evelyne_simoes@hotmail.com

${ }^{6}$ Núcleo de Inovação e Transferência de Tecnologia - NINTEC - Universidade Federal do Piauí - UFPI

Teresina/PI - Brasil mrita@ufpi.edu.br

${ }^{3,7}$ Universidade Federal do Ceará - UFC - Fortaleza/CE - Brasil

lanagrasiela@gmail.com; cpessoa@ufc.br

\section{Resumo}

A espécie Pilocarpus microphyllus Stapf ex Wardlew é uma planta nativa do Norte e Nordeste do Brasil de ocorrência no leste do Estado do Pará, oeste e norte do Maranhão e ao norte do Piauí. É uma das espécies medicinais brasileiras de maior destaque constando em farmacopéias de todo o mundo, devido ao uso de seu princípio ativo pilocarpina. Ao utilizar a base de periódicos Web of Science com o gênero Pilocarpus, o resultado foi de 56 artigos e para a espécie Pilocarpus microphyllus o resultado encontrado foi de 17 artigos. A busca por patentes foi utilizando a base de patentes Derwent Innovation Index, INPI, EPO e USPTO obtendo um resultado contrastante com o número de artigos, considerando o Japão em destaque na proteção da pesquisa envolvendo Pilocarpus. O gênero Pilocarpus com ênfase na espécie Pilcarpus microphyllus por meio dos artigos publicados e das patentes concedidas tanto no Brasil como em outros países destaca a relevante importância econômica e industrial da espécie bem como estabelece perspectivas futuras na descoberta de novas aplicações farmacológicas e biotecnológicas dos outros alcalóides que até o momento permanecem desconhecidas.

Palavras-chave: Jaborandi; Pilocarpus; Pilocarpus microphyllus; Prospecção tecnológica. 


\begin{abstract}
The Pilocarpus microphyllus Stapf ex Wardlew specie is a native plant from north and northeast of Brazil occurring in eastern of Pará state, northwest and north of Maranhão and Piauí. It is one of the most important Brazilian medicinal species presents in pharmacopoeia of the entire world due to the use of its active pilocarpine. When using the Web of Science periodic base with Pilocarpus genus the result was 56 articles and for the specie Pilocarpus microphyllus the result found was 17 articles. The search for patents was using the basic patent Derwent Innovation Index, INPI, EPO and USPTO getting a contrasting result with the number of articles, recital Japan highlighted in protection of research involving Pilocarpus. The genus Pilocarpus with emphasis on Pilocarpus microphyllus specie through published articles and patents granted in Brazil and in other countries highlights the economic and industrial importance of the specie and provides future perspectives in the discovery of new pharmacological and biotechnological applications of the others alkaloids which so far remains unknown.
\end{abstract}

Key-words: Jaborandi; Pilocarpus; Pilocarpus microphyllus; Technological forecasting

\title{
1. Introdução
}

O jaborandi é sinônimo vulgar de várias plantas silvestres do Brasil e países vizinhos, das famílias Piperaceae e Rutaceae, que possuem propriedades medicinais (CORRÊA, 1969). A família Rutaceae possui cerca de 150 gêneros e 1500 espécies (CRONQUIST, 1988). No Brasil se encontram 13 espécies, sendo que 11 delas ocorrem exclusivamente no território brasileiro, com maior ocorrência em sua porção oriental, constituindo o centro de diversidade genética do gênero (SKORUPA, 1996).

A espécie Pilocarpus microphyllus Stapf ex Wardlew é nativa do Norte e Nordeste do Brasil de ocorrência no leste do Estado do Pará, oeste e norte do Maranhão e ao norte do Piauí, movimentando o extrativismo de toneladas todos os anos nestes estados (PINHEIRO, 1997; LIMA, 2008). É uma das espécies medicinais brasileiras de maior destaque constando em farmacopéias de todo o mundo, devido ao uso de seu princípio ativo pilocarpina (LEWIS \& ELVIN-LEWIS, 1977), sendo ainda na atualidade de grande relevância para as indústrias farmacêuticas e farmoquímicas nacionais e internacionais. Das folhas desta espécie são processados os sais cloridrato de pilocarpina, nitrato de pilocarpina, além de sua forma livre (pilocarpina base) utilizados na formulação de colírios para tratamento do glaucoma (TSAI et al., 2003; NORDSTROM et al., 2005; LIMA, 2008), sendo esta patologia de ampla ocorrência mundial e responsável pela exportação deste ativo para Europa, Estados Unidos, Japão, China e Índia movimentando milhões de dólares todos os anos.

Outras aplicações terapêuticas da pilocarpina foram elucidadas posteriormente, como por exemplo, o seu uso na forma oral para o tratamento da xerostomia (BAPTISTA NETO, 2004), mediante tratamento de radioterapia após neoplasias de cabeça e pescoço, sendo aprovado pelo 
FDA (Food and Drug Administration) em 1994 (MOURA, 2003). Atualmente a importância do $P$. microphyllus reside na pesquisa e desenvolvimento da aplicação farmacológica e biotecnológica de outros alcalóides imidazólicos presentes nesta espécie, como recentemente a aplicação do alcalóide epiisopiloturina e epiisopilosina no combate a doenças negligenciadas (VERAS et. al., 2012; PATENTE INPI No PI0904110-9 A2; ROCHA, 2013) e a atividade anti-inflamatória e antinociceptiva do mesmo (SILVA et.al., 2013).

Diante da importância e uso desta espécie, a mesma foi explorada durante décadas no norte e nordeste do Brasil de maneira insustentável, a ponto de atualmente constar na Lista Oficial das Espécies da Flora Brasileira Ameaçadas de Extinção (IBAMA, 2008), sendo de extrema importância iniciativas que agreguem potencial tecnológico que dêem sustentabilidade a espécie devido a relevante importância para diversas comunidades extrativistas de baixa renda no norte e nordeste do Brasil nos estados federativos brasileiros do Piauí, Maranhão e Pará.

O presente trabalho teve como objetivo realizar uma prospecção tecnológica afim de se avaliar o perfil quantitativo das publicações e proteções com o gênero Pilocarpus e da espécie Pilocarpus microphyllus, no indexador Web of Science e a base de dados de patentes, Derwent Innovation Index, Europen patent Office (EPO), no United States Patent and Trademark Office (USPTO) e no banco de dados do Instituto Nacional de Propriedade Industrial (INPI) do Brasil.

\section{Metodologia}

Para levantamento de dados foi utilizada a base de artigos da Web of Science e a base de dados de patentes Derwent Innovation Index, Europen patent Office (EPO), United States Patent and Trademark Office (USPTO) e do Instituto Nacional de Propriedade Industrial (INPI).

O levantamento foi feito em maio de 2014 na base de artigos e patentes, utilizando como palavras-chave os termos utilizando as palavras-chave "Pilocarpus" e "Pilocarpus microphyllus". Os termos em inglês foram utilizados para as bases internacionais, enquanto que os termos em português foram utilizados para a busca de documentos na base nacional, sendo considerados válidos os documentos que apresentassem esses termos no título e/ou resumo. Nesta pesquisa foi utilizado o software Vantage Point para tratamento bibliométrico, geração dos dados quantitativos e identificação de indicadores científicos. Os gráficos de depósitos de patentes que se referem ao ano e países estão com os dados das bases citadas. 


\section{Resultados e Discussão}

Utilizando a base de periódicos Web of Science empregou-se como palavra-chave o gênero Pilocarpus resultando em 56 artigos e refinando para a espécie Pilocarpus microphyllus o resultado encontrado foi de 17 artigos. Os dados levantados por patentes resultaram num total de 97 documentos depositados entre 1918 (ano do primeiro depósito) a 2009. Durante a utilização de busca das patentes foram recuperados apenas os documentos que já tenham sidos publicados (Tabela 1).

Tabela 1. Busca de publicações e patentes por gênero e espécie da planta estudada nas bases de dados.

\begin{tabular}{cccccc}
\hline Palavras-chave & $\begin{array}{c}\text { Artigos }- \text { Web } \\
\text { of Science }\end{array}$ & $\begin{array}{c}\text { Patentes - Derwent } \\
\text { Innovation Index }\end{array}$ & EPO & USPTO & INPI \\
\hline $\begin{array}{c}\text { Pilocarpus } \\
\text { Pilocarpus }\end{array}$ & 56 & 35 & 29 & 4 & 9 \\
microphyllus & 17 & 10 & 5 & - & 5 \\
\hline
\end{tabular}

FONTE: Autoria própria (2013).

A Figura 01 representa os resultados das publicações com o gênero Pilocarpus do período de 1993 a 2012. Sete espécies de Pilocarpus se destacam na busca, P. microphyllus, P. Spicatus, P.jaborandi, P. Pennatifolius, $P$. trachyllophus, P. racemosus e P. riedelianus, dentres estas citadas apenas a espécie Pilocarpus racemosus não ocorre em território brasileiro, sendo encontrada na Venezuela, Colômbia, México, El Salvador, Costa Rica, Cuba, Haiti e Porto Rico (LIMA, 2008).

A espécie $P$. microphyllus se encontra em destaque no número de publicações a partir do ano de 1999 (Figura 1), sendo esta espécie a mais importante do ponto de vista econômico de exploração industrial devido ao elevado teor do alcalóide imidazólico pilocarpina em relação as outras espécies do gênero Pilocarpus de ocorrência nacional (PINHEIRO, 2002). 


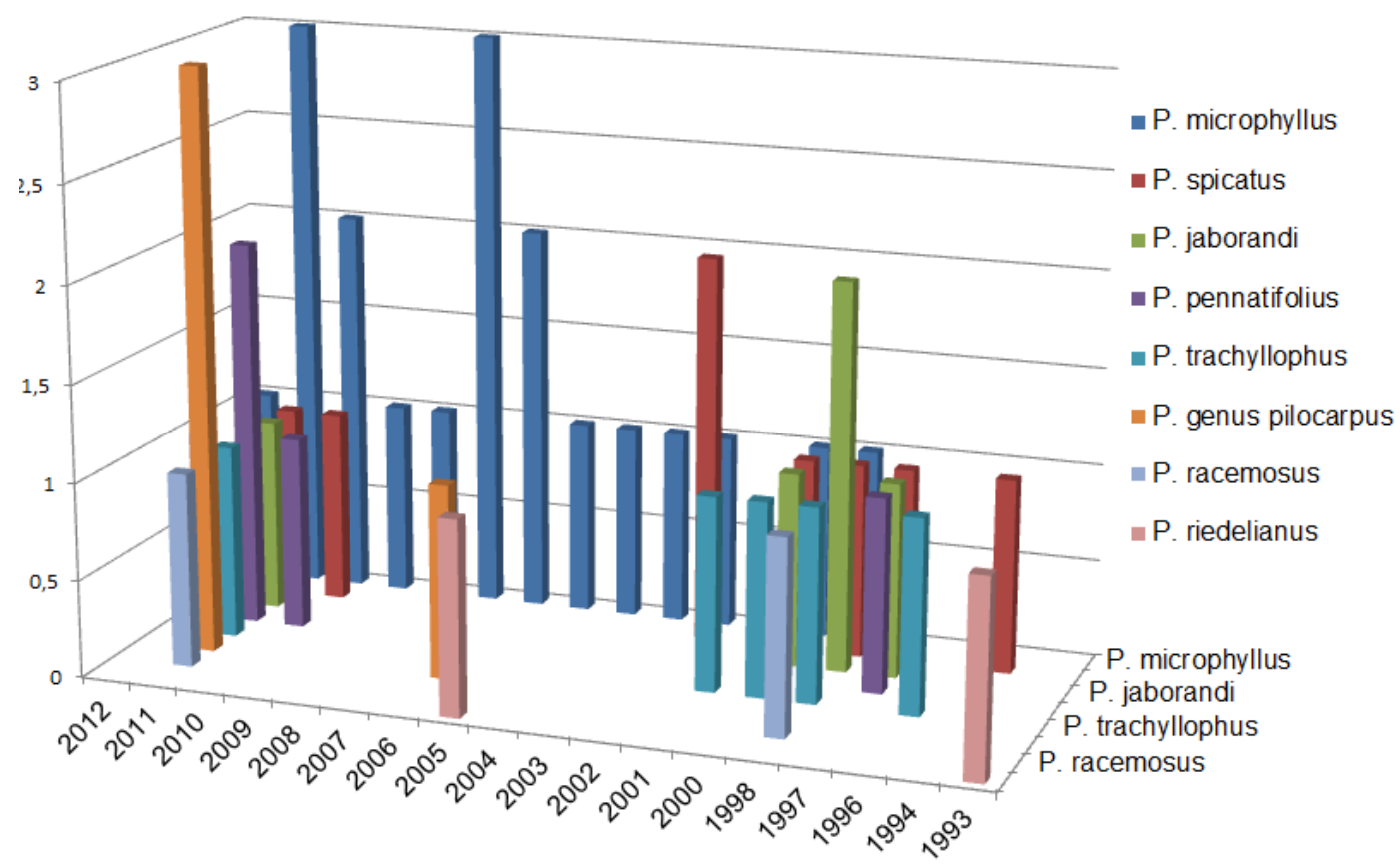

Figura 1 - Número de publicações das principais espécies do gênero Pilocarpus do período de 1993 a 2012 na base de dados da Web fo Science.

FONTE: Autoria própria (2013).

É de grande relevância destacar também que esta espécie $P$. microphyllus foi selecionada dentre as outras para estudos de domesticação e implantação de extensas áreas de cultivo no estado do Maranhão e Piauí, sendo a mesma nativa e não endêmica no Norte e Nordeste do Brasil com registro de ocorrência no Pará, Piauí e Maranhão (PINHEIRO, 2002; LIMA, 2008; ROCHA, 2013). Isto justifica o maior número de publicações de $P$. microphyllus na base de dados Web of science em detrimento as outras espécies do mesmo gênero (Figura 2). 


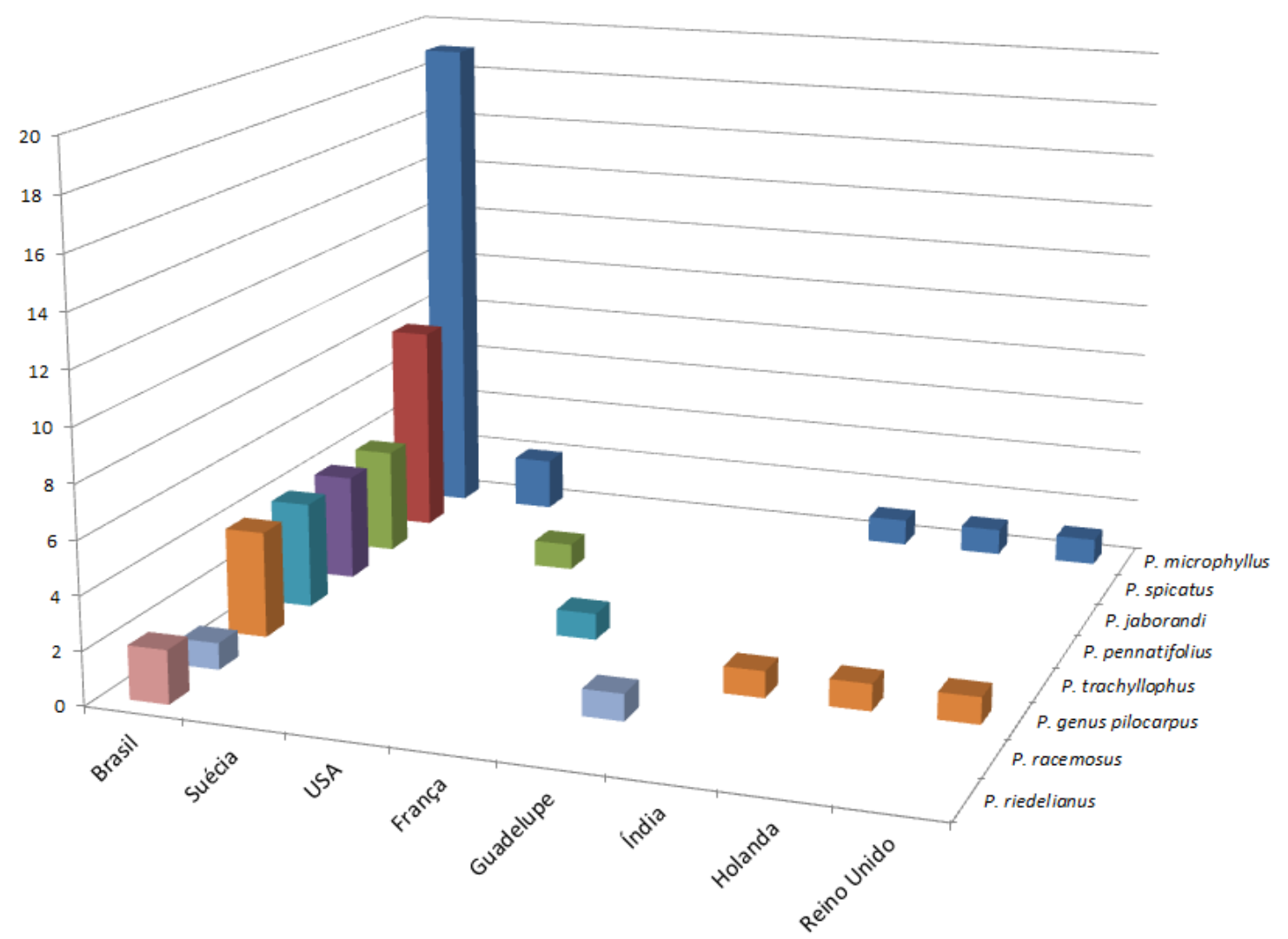

Figura 2 - As publicações das principais espécies da planta Pilocarpus no contexto mundial pesquisado na base de dados Web of Science.

FONTE: Autoria própria (2013).

O Brasil foi o país que mais desenvolveu pesquisas com as diversas espécies de Pilocarpus, por serem amplamente distribuídas em todo território nacional (SKORUPA,1996; ROCHA, 2013). No entanto outros países como Suécia, Índia, Holanda e Reino Unido também realizaram publicações com a espécie $P$. microphyllus (Figura 3).

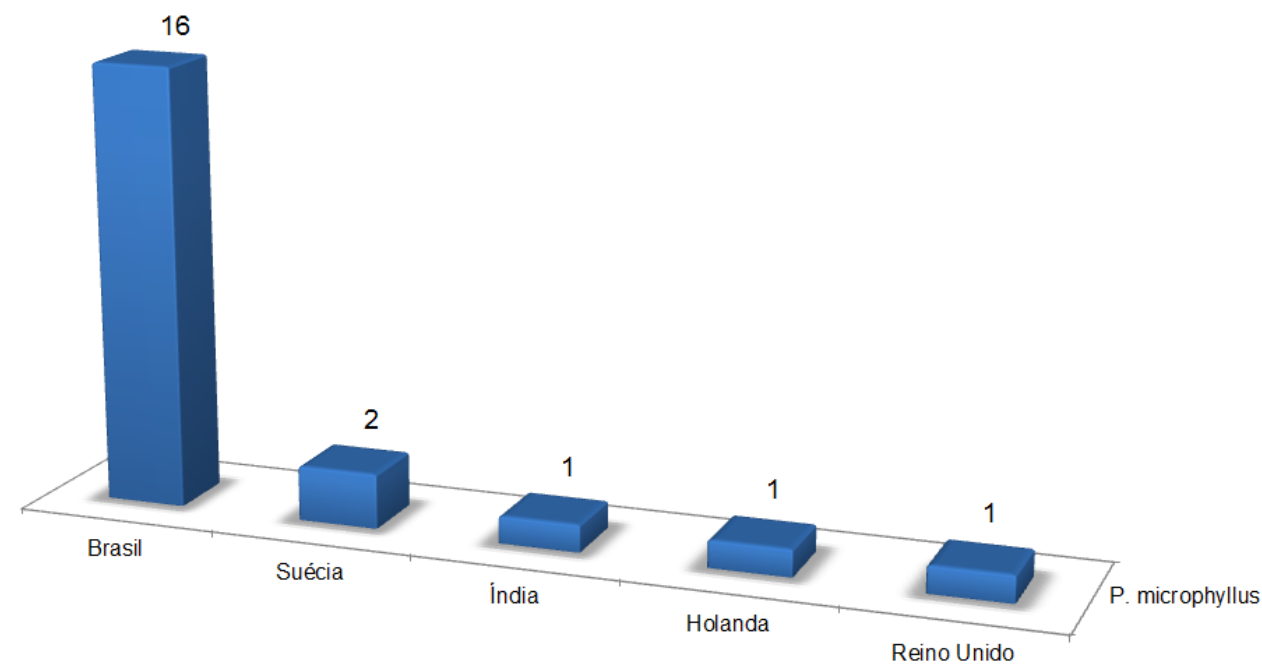

Figura 3 - Participação dos países em artigos publicados na Web of Science (P. microphyllus) do período de 1993 a 2012.

Fonte: Autoria própria (2013). 
Ao verificar as principais áreas nas quais foram publicados os artigos na Web of Science com a espécie $P$. microphyllus, constata-se que a área de Fitotecnia surge em primeiro lugar com 39,38\% das publicações, seguido da área Química e Farmacologia com 26,78\% e 25,00\%, respectivamente (Tabela 2). É possível observar que a maioria dos trabalhos publicados se concentram nas áreas de botânica/agronomia e química destacando principalmente classificações e ocorrência das espécies bem como a identificação e isolamento de constituintes químicos dentre outros. Com relação a área de aplicação biológica/ farmacologia o destaque dos trabalhos está com alcalóide imidazólico de interesse industrial pilocarpina, onde os outros alcalóides permanecem em sua grande maioria inexplorados quanto a aplicação terapêutica.

Tabela 2. Número de publicações e patentes com gênero e espécie da planta estudada.

\begin{tabular}{ccc}
\hline Áreas de Interesse & Registros & \% \\
\hline Fitotecnia & 22 & 39,38 \\
Química & 15 & 26,78 \\
Farmacologia & 14 & 25,00 \\
Bioquímica e Biologia Molecular & 10 & 17,85 \\
Agricultura & 4 & 7,14 \\
Tecnologia de Alimentos & 4 & 7,14 \\
Ciência Ambiental & 3 & 5,35 \\
Medicina Int. Complementar & 3 & 5,35 \\
Outras & 8 & 14,28
\end{tabular}

Fonte: Autoria própria (2013).

A espécie P. microphyllus contém outros alcaloides imidazólicos descritos na literatura com possível potencial farmacológico, biotecnológico e/ou como protótipos na obtenção de novas moléculas, tais como a macaubina, isopilocarpina, epiisopilosina, epiisopiloturina, isopilosina, pilosina e seus isômeros em concentrações variáveis (LUCIO et al., 2000; LORENZI et al., 2002; SANTOS \& MORENO, 2004; BENTO et al., 2010; SAWAYA et.al., 2011; VERAS et al., 2012), dentre os mesmos apenas a epiisopiloturina e epiisopilosina (ROCHA, 2013) apresentam publicações relacionadas a aplicação biológica. A maioria das publicações e patentes se concentram em torno da pilocarpina, suas aplicações no tratamento do glaucoma e xerostomina e a química dos alcalóides (Figura 4). A pesquisa e desenvolvimento de novas aplicações para a P. microphyllus irão agregar maior valor a espécie e consequentemente beneficiará inúmeras comunidades 
extrativistas de baixo poder econômico e IDH (Índice de Desenvolvimento Humano) no Norte e Nordeste do Brasil.

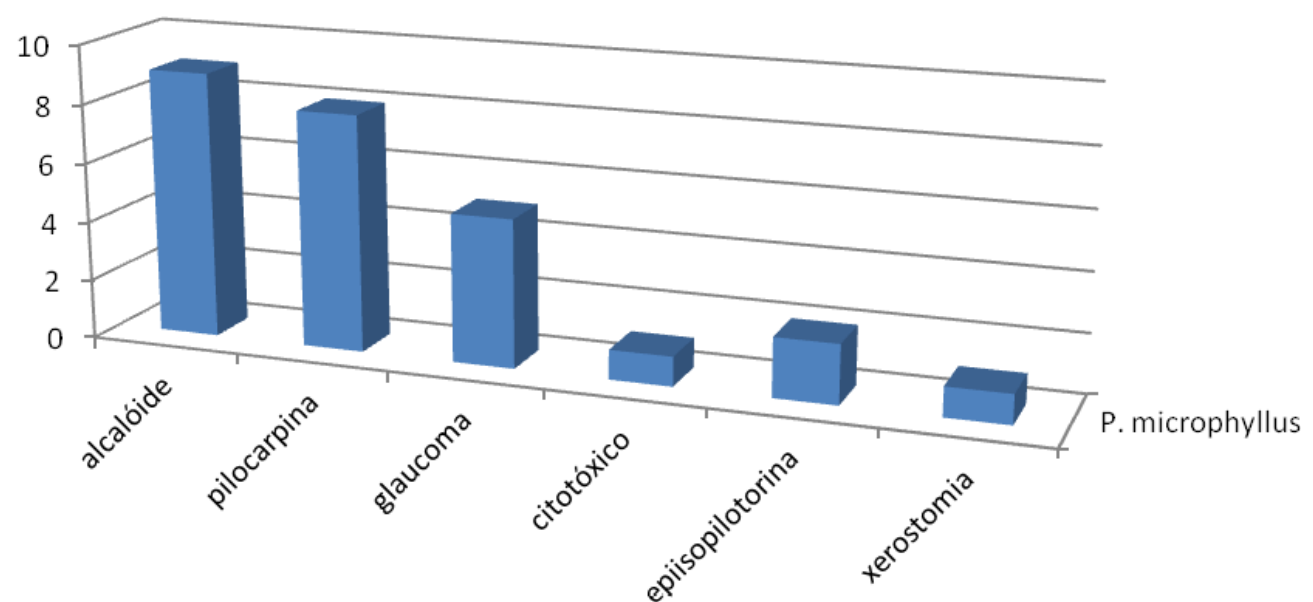

Figura 04. Aspectos tecnológicos mais pesquisados pelos autores com Pilocarpus microphyllus. Fonte: Autoria própria (2013).

Por outro lado, o aumento da produção científica de pesquisadores brasileiros ainda não é suficiente para desencadear impactos importantes para o desenvolvimento econômico no país. O número de patentes depositadas por brasileiros está longe dos números de artigos publicados.

A proteção de pesquisas envolvendo Pilocarpus iniciou em 1918 com a publicação de uma patente e em 1972 com uma patente e quatro anos depois um depósito de uma patente em 1976 ambos do Canadá. Depois de 1987, constatou-se um aumento nos anos seguintes, com uma evolução do número de patentes. O auge foi o depósito de um número expressivo de 18 patentes em 1996 (Figura 5 e 6).

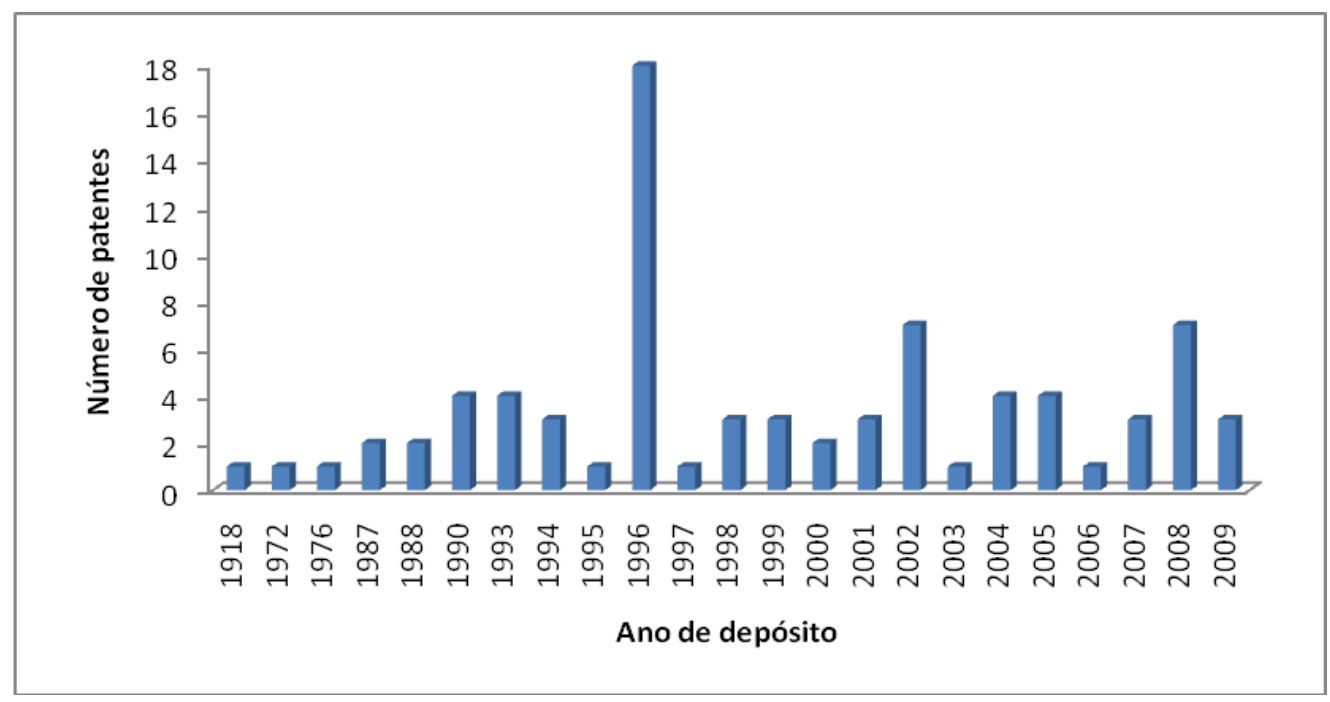

Figura 5 - Número de patentes depositadas por ano para a Pilocarpus.

FONTE: Autoria própria (2013). 
O Brasil ocupa o topo do ranking em número de publicações de artigos em periódicos envolvendo Pilocarpus, com um total de 56 artigos científicos, seguido da Suécia que conta com apenas dois artigos publicados (Figura 2). Este resultado é contrastante com o número de patentes depositadas por país, considerando que o Japão se destaca na proteção da pesquisa envolvendo Pilocarpus, com um número expressivo de patentes de cinco espécies, em detrimento de apenas três espécies da Pilocarpus depositadas no Brasil (Figura 6). Supõe-se, por estes resultados, que o Japão detêm certo pioneirismo, em relação a países emergentes como o Brasil, no que concerne à proteção de sua pesquisa.

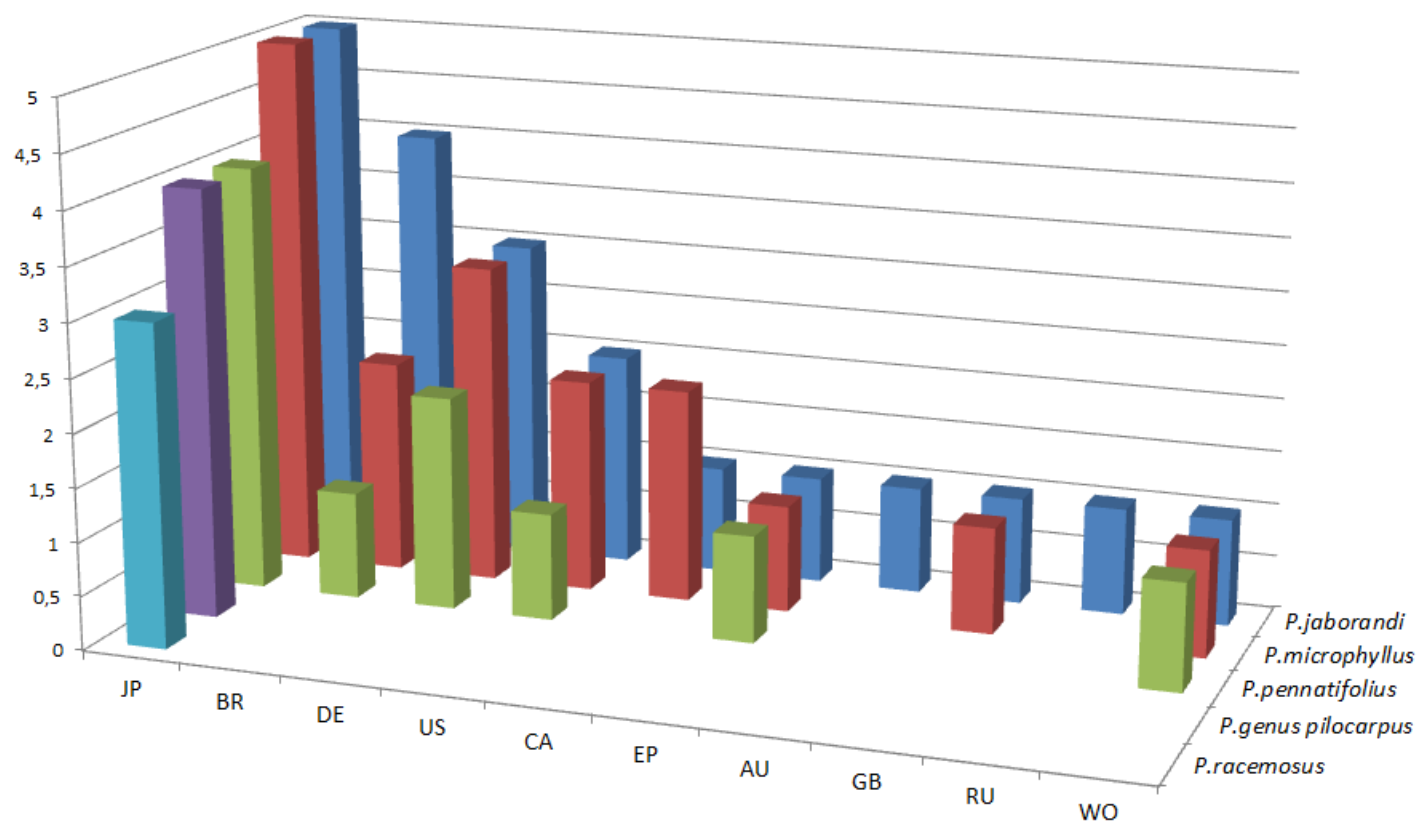

Figura 6 - Países que mais depositaram patentes envolvendo Pilocarpus.

FONTE: Autoria própria (2013).

O Brasil possui baixa competitividade e tem criado pouco esforço em inovar na área de produtos tecnológicos, possivelmente deve-se à imaturidade do sistema de inovação (articulação eficiente entre governo, empresas e universidades, capaz de promover um sistema de P \& D), o Brasil não possui uma produção expressiva nas espécies de Pilocarpus refletindo ainda mais à falta de cultura de proteger as tecnologias desenvolvidas por meio da propriedade industrial (Figura $6 \mathrm{e}$ 7). 


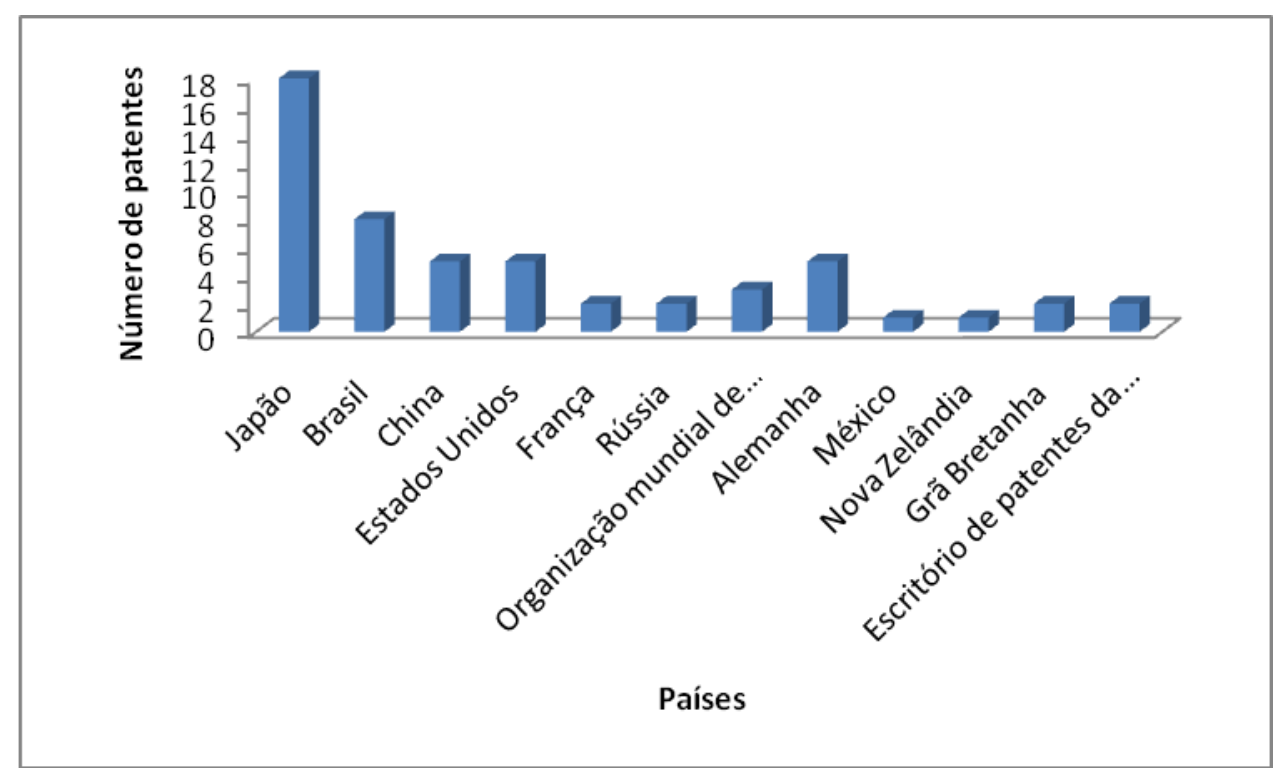

Figura 7 - Países que mais patentearam envolvendo Pilocarpus microphyllus. FONTE: Autoria própria (2013).

As Figuras 8 e 9 apresentam os códigos da classificação internacional de patentes patentes da base Derwent Innovation Index e do INPI, EPO e USPTO, respcetivamente, limitam-se nos primeiros 4 dígitos. Para se ter uma visão geral das diferentes áreas de pesquisa e aplicação, o código A61K é o que mostrou uma maior ocorrência e refere-se a especificamente a preparações para finalidades médicas, odontológicas ou higiênicas. Nesta mesma classe o código A61P direciona-se a atividades terapêuticas de compostos químicos ou de preparação medicinais.

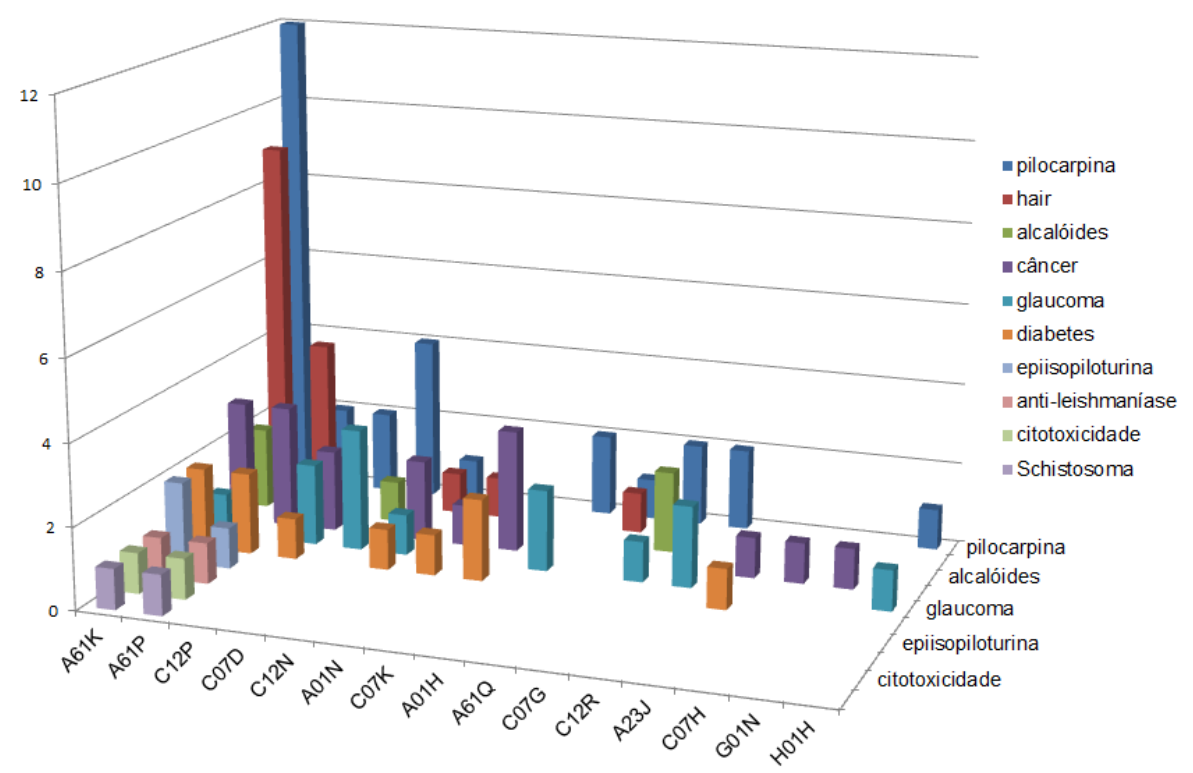

Figura 8 - Patentes por código de classificação internacional de acordo com o potencial tecnológico. FONTE: Autoria própria (2013). 


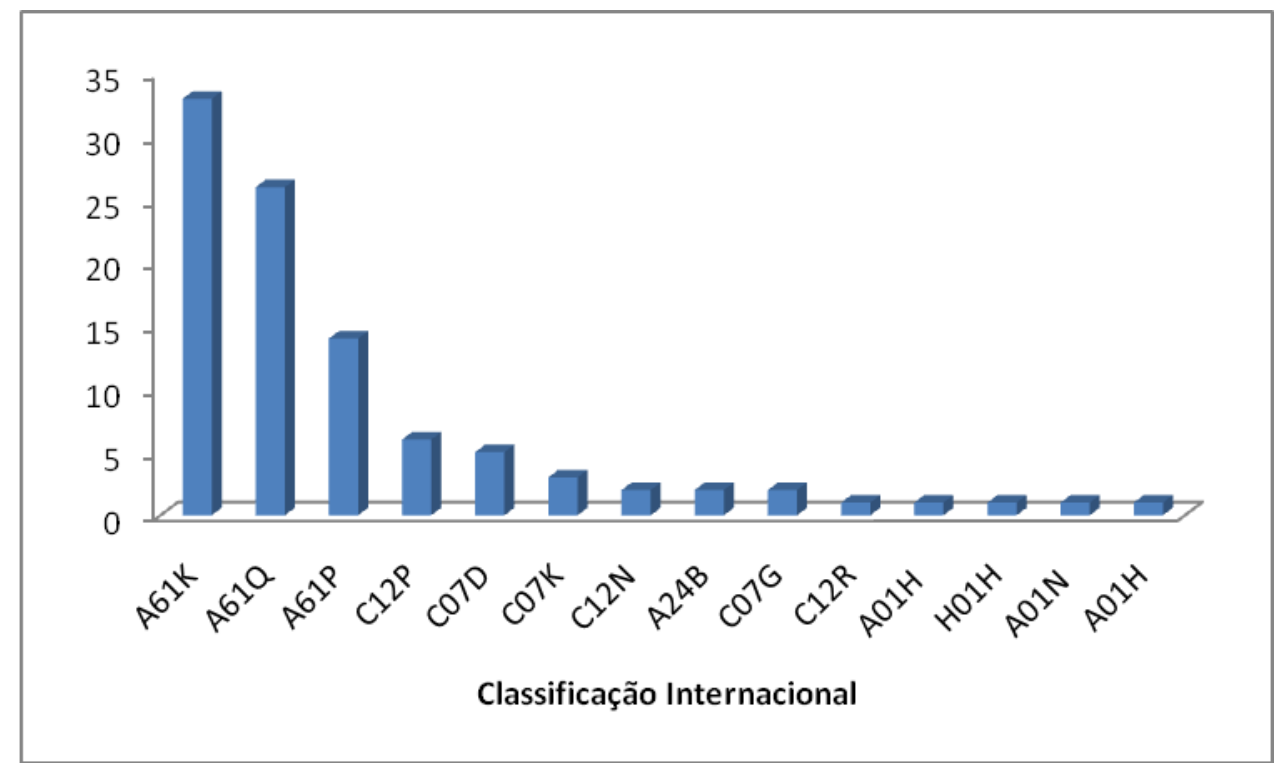

Figura 9 - Patentes por código de classificação internacional de acordo com o potencial tecnológico. FONTE: Autoria própria (2013).

\section{Conclusão}

O estudo de prospecção tecnológica com o gênero Pilocarpus com ênfase na espécie Pilcarpus microphyllus por meio dos artigos publicados e das patentes concedidas tanto no Brasil como em outros países destacou a relevante importância mundial desta espécie como fonte do alcalóide pilocarpina utilizado a décadas no tratamento do glaucoma e xerostomia, no entanto as perspectivas futuras com a espécie residem no fato da descoberta de novas aplicações farmacológicas e biotecnológicas dos outros alcalóides que até o presente momento permanecem em sua grande maioria desconhecidas e inexploradas. O incentivo por parte do Governo Federal em apoiar pesquisas relacionadas a espécies nativas brasileiras, principalmente as de ocorrência em áreas de menor renda per capita e IDH (Índice de Desenvolvimento Humano) como no Norte e Nordeste do Brasil seriam de extrema importância para agregar valor a espécie e estimular o desenvolvimento econômico destas regiões.

Este estudo nos revela também que as universidades brasileiras têm potencial de geração de novas tecnologias passíveis de patenteamento e desenvolvimento de produtos, os resultados apontaram 56 artigos indexados em relação aos outros países que apresentaram menor publicações. Esses valores em números no Brasil são bastante relevantes em relação às publicações, porém indicam um gargalo nos registros das patentes. 
Agradecimentos:

CNPq, CAPES, FUNCAP

\section{Referências Bibliográficas}

BAPTISTA NETO, C. SUGAYA, N.N. Tratamento da xerostomia em pacientes irradiados na região da cabeça e do pescoço. Revista Biociência, Taubaté, v.10, n.3, p.147-151, jul./set. 2004.

BENTO, R.R.F.; SILVA, L.E. da.; FARIA, J.L.B.; FREIRE, P.T.C.; DE OLIVEIRA, M.C.F.; ROMERO, N.R.; TEIXEIRA, A.M.R.; PONTES, F.M.,. Comparative vibrational spectra of pilosine and epiisopilosine crystals. Brazilian Journal of Physics 40, 217-223, 2010.

CORRÊA, M.P. Dicionário das plantas úteis do Brasil, Vol. 4, Ministério da Agricultura, Rio de Janeiro. p. 360-369, 1969.

CRONQUIST, A. Na integrated system of classification of flowering plants. New York, Columbia University Press, 1981. 1262p.

CRONQUIST, A. The Evolution and Classification of Flowering Plants, 2. ed. The New York Botanical Garden, New York, 1988, 555p.

IBAMA. (Instituto Brasileiro do Meio Ambiente e dos Recursos Naturais Renováveis). Lista oficial de Espécies Brasileiras Ameaçadas de Extinção. Instrução Normativa MMA n06, de 26 de setembro de 2008.

LEWIS, W.H.; ELVIN-LEWIS, M.P.F. Medical botany - plants affecting man's health. New York: John Wiley \& Sons. 1977. 515p.

LIMA, D. F. Alcalóides de interesse industrial: aspectos químicos e biológicos. 2008. $56 \mathrm{f}$. Monografia (Especialização em Plantas Medicinais) - Pós-Graduação em Plantas Medicinais, Universidade Federal de Lavras, Lavras, 2008.

LORENZI, H.A.; MATOS, F.J. Plantas Medicinais no Brasil: Nativas e Exóticas Cultivadas. Instituto Plantarum, Nova Odessa, São Paulo, 2002.

LUCIO, E.M.R.A.; ROSALEN, P.L.; SHARAPIN, N.; SOUZA BRITO, A.R.M. Avaliação toxicológica aguda e screening hipocrático da epiisopilosina, alcaloide secundário de Pilocarpus microphyllus Stapf. Revista Brasileira de Farmacognosia 9, 23-35, 2000.

MARQUES, M.E.T.; COSTA, J.P.C. Jaborandi (Pilocarpus microphillus). Belém: EMBRAPACPATU, 1994. 4 p. (Recomendações Básicas, 27).

MOURA, E.F. Divergência genética entre acessos de jaborandi (Pilocarpus microphyllus). 2003. 75f. Dissertação (Mestrado em Genética e Melhoramento de Plantas) - Programa de PósGraduação em Genética e Melhoramento de Plantas, Universidade Federal de Lavras, Lavras, 2003. 
NORDSTROM, B.L.; FRIEDMAN, D.S.; MOZAFFARI, E.; QUIGLEY, H.A.; WALKER, A.M. Persistence and adherence with topical glaucoma therapy. American Journal of Ophthalmology, v. 140, n. 4 , p. 598-606. 2005.

PINHEIRO, C.U.B. Jaborandi (Pilocarpus sp., Rutaceae): uma espécie selvagem e sua rápida transformação em cultivar. Economic Botany. v. 51, n. 1, p.49-58, 1997.

PINHEIRO, C.U.B. Extrativismo, cultivo e privatização do jaborandi (Pilocarpus microphyllus ex Holm., Rutaceae) no Maranhão, Brasil. Acta Botânica Brasilica. São Paulo, v. 16, n.2, abril 2002.

ROCHA, J.A. Variabilidade genética e avaliação anti-schistosoma dos alcalóides pilosina, epiisopilosina, isopilosina e macaubina de jaborandi (Pilocarpus microphyllus Stapf ex Wardlew.). 2013. 145f. Dissertação (Mestrado em Biotecnologia) - Programa de Pós-Graduação em Biotecnologia - Universidade Federal do Piauí, Parnaíba, 2013.

SANTOS, A. P.; MORENO, P. R. H. Pilocarpus spp.: A survey of its chemical constituents and biological activities. Brazilian Journal of Pharmaceutical Sciences, v.40, n. 2, p. 115 - 137. 2004.

SAWAYA, A.C.H.F.; VAZ, B.G.; EBERLIN, M.N.; MAZZAFERA, M. Screening species of Pilocarpus (Rutaceae) as sources of pilocarpina and other imidazole alkaloids. Genetic Resources and Crop Evolution. V.58, p. 471-480, 2011.

SILVA, V.G.; SILVA, R.O.; DAMASCENO, S.R.B.; ARAGÃO, K.S.; GUIMARÃES, M.A.; CAMPOS,S.A.; VERAS, L.M.C.; GODEJOHANN, M.; LEITE, J.R.S.A.; BARBOSA, A.L.R.; MEDEIROS, J.V.R. Anti-inflammatory and antinociceptiva activity of epiisopiloturina, an imidazole alkaloid isolated from Pilocarpus microphyllus. Journal of Natural Products.The American Chemical Society and American Society of Pharmacognosy, 2013.

TSAI, J.C.; MCCLURE, C.A.; RAMOS, S.E.; SCHLUNDT, D.G.; PICHERT, J.W. Compliance barriers in glaucoma: a systematic classification. Journal of Glaucoma, v. 12, n. 5, p. 393-398. 2003.

VEGEFLORA, Plano de manejo florestal comunitário: Pilocarpus microphyllus Stapf ex Wardi. Parnaíba, VEGEFLORA, 2010. 71 p.

VERAS, L.M.; GUIMARÃES, M.A.; CAMPELO, Y.D.; VIEIRA, M.M.; NASCIMENTO, C.; LIMA, D.F.; VASCONCELOS, L.; NAKANO, E.; KUCKELHAUS, S.S.; BATISTA, M.C.; LEITE, J.R.; MORAES, J. Activity of epiisopiloturine Against Schistosoma mansoni. Current Medicinal Chemistry, v. 19, p. 2051-58, 2012.

Recebido: 22/06/2014

Aprovado: 22/02/2015 